\title{
Optimal Quantum Pumps
}

\author{
J. E. Avron ${ }^{(a)}$, A. Elgart ${ }^{(b)}$, G.M. Graf ${ }^{(c)}$ and L. Sadun ${ }^{(d)}$ \\ (a) Department of Physics, Technion, 32000 Haifa, Israel \\ (b) Department of Physics, Jadwin Hall, Princeton University, Princeton, NJ 08544, USA \\ (c) Theoretische Physik, ETH-Hönggerberg, 8093 Zürich, Switzerland \\ (d) Department of Mathematics, University of Texas, Austin Texas 78712, USA
}

\begin{abstract}
We study adiabatic quantum pumps on time scales that are short relative to the cycle of the pump. In this regime the pump is characterized by the matrix of energy shift which we introduce as the dual to Wigner's time delay. The energy shift determines the charge transport, the dissipation, the noise and the entropy production. We prove a general lower bound on dissipation in a quantum channel and define optimal pumps as those that saturate the bound. We give a geometric characterization of optimal pumps and show that they are noiseless and transport integral charge in a cycle. Finally we discuss an example of an optimal pump related to the Hall effect.
\end{abstract}

PACS numbers: 72.10.Bg, 73.23.-b

Introduction: A time dependent scatterer can transport charges between electron reservoirs which otherwise are in thermal equilibrium. This makes it into a quantum pump. An example is shown in Fig. 11 where the flux $\Phi$ is slowly time dependent. While operating, the pump may also generate (excess) noise in the ideal channels connecting to the reservoirs and dissipate energy in the reservoirs. A pump is adiabatic when its frequency $\omega$ is slow compared with the natural time scale $\tau$ of the scattered electrons, e.g., the Wigner time delay [1]. The adiabaticity parameter, $\varepsilon=\omega \tau \ll 1$, plays the role analogous to the semiclassical limit: A particle localized in energy is scattered at a well defined time as measured by the pump cycle.

The theory of adiabatic pumps is concerned with a description of transport and noise in terms of the the frozen, on-shell, scattering matrix, $\mathcal{S}(t ; E)$. In the case that the pump is connected to say, two reservoirs, as in Fig, 1, via $n \geq 2$ channels, $\mathcal{S}$ is an $n \times n$ unitary matrix parameterized by the frozen time $t$ and energy $E$. At low temperatures, $\tau / \beta \ll \hbar$, the transport and noise are determined by the electrons near the Fermi energy, $\mu$, and hence by $\mathcal{S}(t ; \mu)$.

Two basic results in the theory of adiabatic pumps are Eq. (3) below, originally due to [2,3], for the instantaneous expected current in a channel and a formula of Levitov and Lesovik, [4], for the current correlations, and the noise, in a pump cycle. Here we focus on further quantities that admit a local description in time, like dissipation at $\beta=\infty$, which is related to integral transport, and entropy and noise production at $\beta<\infty$.

Energy shift: It turns out that instantaneous response is determined by the energy shift matrix, $\mathcal{E}$. It is conjugate to Wigner time delay, $\mathcal{T}(t, \mu)=$ $-i \hbar \partial_{\mu} \mathcal{S}(t, \mu) \mathcal{S}^{\dagger}(t, \mu)$, and is defined by:

$$
\mathcal{E}(t, \mu)=i \hbar \partial_{t} \mathcal{S}(t, \mu) \mathcal{S}^{\dagger}(t, \mu) .
$$

It is of order of the adiabaticity parameter $\varepsilon$ with matrix elements:

$$
\mathcal{E}_{j k}=i \hbar\left\langle\psi_{k} \mid \dot{\psi}_{j}\right\rangle,
$$

where $\left|\psi_{j}\right\rangle$ is the $j$-th row of the scattering matrix. For example, the diagonal matrix elements of $\mathcal{E}$ determine the instantaneous net current entering the reservoir through the $j$-th channel (even at zero temperature)

$$
\dot{Q}_{j}=\frac{e}{h} \mathcal{E}_{j j}+O\left(\varepsilon^{2}\right) .
$$

(A formal derivation of this result follows easily from the analog of Eq. (11) and Eq. (12) below.) As we shall see, the energy shift also provides information on dissipation and noise, and leads to a characterization of optimal pumps.

Lower bound on dissipation: In order to motivate the notion of optimal pump we shall first establish a lower bound on the dissipation in a quantum channel.

Suppose a reservoir at zero temperature is connected by a channel to a general time independent, possibly nonthermal, particle source. (In the applications we shall consider, this particle source will be an adiabatic quantum pump.) Let $\dot{E}$ denote the net energy flux (power) and $\dot{Q}$ the charge flux (current), flowing out from the source to the cold reservoir. The power dissipated in the channel, i.e. the difference between the energy flow and the energy flow that can be recovered, satisfies the general lower bound:

$$
\dot{E}-\frac{\mu}{e} \dot{Q} \geq \frac{R_{k}}{2} \dot{Q}^{2},
$$

with $R_{k}=h / e^{2}$ the (von Klitzing) unit of resistance. This bound does not depend on the nature of the particle source. It is a consequence of the fact that a channel is one dimensional, that the charge carriers are noninteracting (spinless) fermions, that the reservoir is at 
zero temperature and that the particle source is time independent. A source that saturates the lower bounds Eq. (4) will be called optimal.

Proof: A time-independent particle source connected to a channel is characterized by the filling $0 \leq n(k) \leq 1$ of states at momentum $k$. Let $\dot{E}(\dot{Q})$ be the energy (charge) current out of the source and $\epsilon(k)$ the dispersion relation. Then,

$$
\begin{aligned}
& \dot{Q}=\frac{e}{2 \pi \hbar} \int_{\epsilon^{\prime}(k)>0} d k n(k) \epsilon^{\prime}(k), \\
& \dot{E}=\frac{1}{2 \pi \hbar} \int_{\epsilon^{\prime}(k)>0} d k n(k) \epsilon(k) \epsilon^{\prime}(k) .
\end{aligned}
$$

The bathtub principle (w.r.t. the measure $\epsilon^{\prime}(k) d k$ on $\left\{k \mid \epsilon^{\prime}(k)>0\right\}$; see e.g., [5]) states that for fixed $\dot{Q}$ the quantity $\dot{E}$ is minimized by $n(k)=\theta(\mu-\epsilon(k))$ for some $\mu$. Assuming $k \epsilon^{\prime}(k) \geq 0, \epsilon(0)=0$, we find for the minimizer

$$
\dot{Q}=\frac{e \mu}{2 \pi \hbar}, \quad \dot{E}=\frac{\mu^{2}}{4 \pi \hbar} .
$$

This implies that

$$
\dot{E} \geq \frac{R_{k}}{2} \dot{Q}^{2}
$$

with equality holding if and only if the source is a reservoir at thermal equilibrium at zero temperature so that the carriers fill the Fermi sea up to energies $\mu$.

Consider next a channel connecting a particle source, denoted by subscript + , to a thermal reservoir, denoted by subscript - at zero temperature and with chemical potential $\mu_{-} . \quad \dot{E}=\dot{E}_{+}-\dot{E}_{-}\left(\dot{Q}=\dot{Q}_{+}-\dot{Q}_{-}\right)$ is the net energy (charge) flowing into the cold reservoir. Since the reservoir is at zero temperature, $\dot{E}_{-}=$ $\frac{R_{k}}{2} \dot{Q}_{-}^{2}=\frac{\mu_{-}^{2}}{2 h}$. Meanwhile, $\dot{E}_{+}$is at least $\frac{R_{k}}{2} \dot{Q}_{+}^{2}$. Hence $\dot{E} \geq \frac{R_{k}}{2}\left(\dot{Q}_{+}^{2}-\dot{Q}_{-}^{2}\right)=\frac{R_{k}}{2} \dot{Q}^{2}+\frac{\mu_{-}}{e} \dot{Q}$. Equality occur if and only if the source also fills a Fermi sea up to some energy $\mu_{+}$. Eq. (4) bounds the dissipation in an ideal channel.

Optimal pumps: An adiabatic pump approximates a time-independent (and typically non-thermal) particle source that connects to the $n$ channels. The lower bound, Eq. (4), motivates the following definition: We say that an adiabatic pump is optimal if the bound Eq. (4) is saturated for all times and all channels in the adiabatic limit. Since the left hand side of Eq. (4) is made of two terms that are each of order $\varepsilon$ while the right hand side is a term of order $\varepsilon^{2}$, saturating the bound means that equality of the two sides holds to order $\varepsilon^{2}$.

For the $j$-th channel of an adiabatic pump connected to reservoirs at zero temperature we shall establish:

$$
\dot{E}_{j}-\frac{\mu}{e} \dot{Q}_{j}=\frac{1}{2 h} \sum_{k}\left|\mathcal{E}_{j k}\right|^{2}+O\left(\varepsilon^{3}\right) .
$$

It follows from Eq. (3) and Eq. (4) that the $j$-th channel is optimal if $\mathcal{E}_{j k}=0$ for all $k \neq j$. This leads to a simple criterion for optimal pumps: A pump is optimal if and only if the energy shift $\mathcal{E}$ is a diagonal matrix for all times.

The notion of optimal pumps is geometric. This is seen from the fact that the vanishing condition on matrix elements of the energy shift, Eq. (2), is invariant under reparameterization of time.

For $n$-channel scattering, the space of Hermitian matrices $\mathcal{E}$ has $n^{2}$ real dimensions, while the space of diagonal matrices is $n$ dimensional. In particular, pumps with a single channel are automatically optimal. Below we shall give an example of an optimal pump with $n=2$ that models the quantum Hall effect.

The scattering matrix associated with a diagonal energy shift is $\mathcal{S}(t)=U_{d}(t) \mathcal{S}_{0}$ where $U_{d}(t)$ is a diagonal unitary and $\mathcal{S}_{0}$ a constant unitary matrix. According to [6], this special form of an $\mathrm{S}$ matrix turns out to characterize of noiseless pumps in the theory of Levitov and Lesovik. Optimal pumps are therefore distinguished in more than one way.

Integral charge transport: Optimal channels transport integral charge in a cycle of the pump. This can be seen from Eq. (3) for the $j$-th channel:

$$
\frac{Q_{j}}{e}=-\frac{i}{2 \pi} \int\left\langle\psi_{j} \mid \dot{\psi}_{j}\right\rangle d t=-\frac{i}{2 \pi} \oint\left\langle\psi_{j} \mid d \psi_{j}\right\rangle
$$

For an optimal channel $\left|\dot{\psi}_{j}\right\rangle$ is parallel to $\left|\psi_{j}\right\rangle$, since $\left\langle\psi_{k} \mid \dot{\psi}_{j}\right\rangle=0$, hence it can only accumulate a phase along the path. Since $\left|\psi_{j}\right\rangle$ is single valued, the total phase accumulated on a closed path must be an integer multiple of $2 \pi$. It is worth remarking that in this case Eq. (9) not only expresses the expectation value of the charge transport in a cycle, as it does by virtue of its derivation, but also the actual charge transport, since the stated condition implies absence of noise, i.e., vanishing variance.

The right hand side of Eq. (9) shows that charge transport is geometric: It depends on the path but is independent of its parameterization. In spite of this geometric interpretation, the charge transport is not quantized in the sense that it has no topological content: A small deformation of the scattering matrix will be a deformation away from optimality and will deform the charge transport away from the integers.

Geometric interpretation: The dissipation formulas admit a geometric interpretation. $\left|\psi_{j}\right\rangle$, being normalized, can be viewed as a point on the sphere $S^{2 n-1} \subset \mathbb{C}^{n}$. The Hopf map [7] $\pi: S^{2 n-1} \rightarrow \mathbb{C} P^{n-1}$, which 'forgets' the phase of $|\psi\rangle$, turns $S^{2 n-1}$ into a fiber (circle) bundle with base space $\mathbb{C} P^{n-1}$. The $j$-th row of $\mathcal{E}$ describes the velocity of $\left|\psi_{j}\right\rangle$ in $S^{2 n-1}$. Of this, $\mathcal{E}_{j j}$ is the projection of this velocity onto the fiber — the changing phase of $\left|\psi_{j}\right\rangle$ — while the matrix elements $\mathcal{E}_{j k}$, with $k \neq j$, give the projection of this velocity onto $\mathbb{C} P^{n-1}$. The current 
$\dot{Q}_{j}$, and the minimal dissipation $\left|\mathcal{E}_{j j}\right|^{2} / 4 \pi$, are both functions of motion in the fiber, while the excess dissipation is the "energy" (that is, squared velocity) associated with motion in the base.

Entropy and noise: So far we did not consider correlations between the current (or power) at different times. Entropy and noise production involve such correlations. The analysis depends critically on which of the two small parameters, $\hbar \omega$ and $\beta^{-1}$, is smaller.

If $\hbar \omega \ll \beta^{-1} \ll \hbar / \tau$ (e.g., in the experiment of Switkes et. al. [8]), then the correlations between current (and power) at different portions of a cycle are negligible, and one can meaningfully speak of the entropy (noise) production per unit time $\dot{S}_{j}\left(\dot{N}_{j}\right)$ in the $j$-th channel. We remark, without proof, that these quantities too are determined by the energy shift and are proportional to the excess energy dissipation:

$$
\dot{S}_{j}=\frac{\beta}{4 \pi \hbar} \sum_{k \neq j}\left|\mathcal{E}_{j k}\right|^{2}, \quad \dot{N}_{j}=\frac{\beta e^{2}}{12 \pi \hbar} \sum_{k \neq j}\left|\mathcal{E}_{j k}\right|^{2} .
$$

If $\beta^{-1} \ll \hbar \omega$, however, then the analysis is fundamentally nonlocal in time, and one is naturally lead to study the noise or entropy generated by a complete cycle, as in 叫.

Dissipation in adiabatic pumps: To derive Eq. (8), we note that the left hand side of Eq. (8) can be written as

$$
\dot{E}_{j}-\frac{\mu}{e} \dot{Q}_{j}=\frac{1}{2 \pi \hbar} \int d E(E-\mu)\left(n_{+j}(E)-n_{-}(E)\right) .
$$

where $n_{-}(E)=\theta(E-\mu)$ is the distribution of the electrons that arrive from the (zero temperature) reservoirs and $n_{+j}(E)$ is the distribution of the electrons that enter the $j$-th reservoir. The pump scrambles the incoming distribution and produces a (non-thermal) outgoing distribution $n_{+j}(E)$. Calculating the outgoing distribution is a problem in adiabatic scattering theory.

We will show that the outgoing density at energy $E$, on the $j$-th channel, is

$$
\begin{aligned}
\left(n_{+}(E)\right)_{j j} \approx & \theta(E-\mu)+\left(\mathcal{E}_{j j}+O\left(\varepsilon^{2}\right)\right) \delta(E-\mu) \\
& -\left(\mathcal{E}^{2}\right)_{j j} \delta^{\prime}(E-\mu) / 2,
\end{aligned}
$$

where $\approx$ means that the operator on the left hand side can be approximated by the the semi-classical distributions (symbol) on the right hand side to second order in $\varepsilon$. Inserting this in Eq. (11), and using the fact that $\left(\mathcal{E}^{2}\right)_{j j}=\sum_{k=1}^{n}\left|\mathcal{E}_{j k}\right|^{2}$, gives Eq. (8). This relation establishes an instantaneous identity between charge and energy transport (which are first order) and dissipation (which is second order). This is remarkable because $\mathcal{S}$ gives only the leading adiabatic approximation to the scattering matrix and is therefore inadequate to describe general second order processes and first order processes beyond the leading behavior. In particular, neither $\dot{E}$ nor $\dot{Q}$ are correctly computable to second order in $\varepsilon$ but Eq. (8) is. Eq. (3) is derived along similar, though somewhat simpler, lines.

Adiabatic scattering: It remains to derive Eq. (12). We start with the standard point of view [9] of timedependent scattering theory, which views the S-matrix as a comparison of a "free" dynamics, generated by $H_{0}$, and the interacting dynamics, generated by $H(t)$. For example, we can pick for $H_{0}$ the Hamiltonian associated with disconnected channels. Let $U(t, s)$ and $U_{0}(t, s)$ denote the corresponding evolutions. Then, the S-matrix is defined by the limit (which we assume exists)

$$
\mathcal{S}_{d}(t)=\lim _{T \rightarrow \infty} U_{0}(t, T) U(T,-T) U_{0}(-T, t) .
$$

In the absence of a scatterer, $U=U_{0}$ and $\mathcal{S}$ is the identity, as it should be. When the scatterer is time independent, $H(t)=H$, the existence of the $T \rightarrow \infty$ limit for the factors $U(0,-T) U_{0}(-T, t)$ and $U_{0}(t, T) U(T, 0) \mathrm{im}-$ plies that $\mathcal{S}_{d}(t)$ is independent of $t$, as it should.

$\mathcal{S}_{d}(t)$ maps incoming wave packet to outgoing ones. When applied to a wave packet near the scatterer, it describes the mapping for an incoming wave, originating at the reservoirs, that hits the scatterer at time $t$.

It follows from Eq. (13) that, provided the limit exists,

$$
i \hbar \dot{\mathcal{S}}_{d}(t)=\left[H_{0}, \mathcal{S}_{d}(t)\right] .
$$

This says that the free dynamics controls when a wave packet hits the scatterer. Eq. (14) determines $n_{+}\left(H_{0}\right)$ to be

$$
\mathcal{S}_{d}(t) n_{-}\left(H_{0}\right) \mathcal{S}_{d}(t)^{\dagger}=n_{-}\left(H_{0}-i \hbar \dot{\mathcal{S}}_{d}(t) \mathcal{S}_{d}(t)^{\dagger}\right) .
$$

In the time independent case, $\dot{\mathcal{S}}_{d}=0$, and Eq. (15) is an expression of conservation of energy. For time dependent scattering, Eq. (15), describes the scattering out of the energy shell. This is the motivation for calling $i \hbar \dot{\mathcal{S}}_{d}(t) \mathcal{S}_{d}(t)^{\dagger}$ the operator of energy shift.

In the adiabatic limit, the (exact) time dependent scattering matrix $\mathcal{S}_{d}(t)$ can be approximated by the time independent scattering matrix for the scatterer frozen at the time of the scattering $t$. Namely, $\mathcal{S}_{d}(t) \approx$ $\delta\left(\mu-\mu^{\prime}\right) \mathcal{S}(t ; \mu)$, with $\mathcal{S}(t ; \mu)$ the on-shell scattering matrix of Eq. (1). Similarly, the operator of energy shift, $i \hbar \dot{\mathcal{S}}_{d}(t) \mathcal{S}_{d}(t)^{\dagger}$, can be approximated in terms of the onshell matrix of energy shift, $\mathcal{E}(t, \mu)$, of Eq. (11). Inserting this in Eq. (15) gives Eq. (12) in the semiclassical limit.

An optimal pump: An example of an optimal pump is shown schematically in Fig. 1. Each one of the two channels is connected to a reservoir at zero temperature on one end and to the loop of circumference $\ell$ on the other. The loop is threaded by a time dependent magnetic flux $\Phi$, which is the engine of the pump. The 
scattering at the vertices is a permutation matrix corresponding to the arrows in the figure. The frozen scattering matrix of the pump is therefore diagonal, with the phases of the two reflection coefficients determined by $\Phi$ :

$$
r=e^{i(k \ell+\Phi)}, \quad r^{\prime}=e^{i(k \ell-\Phi)} .
$$

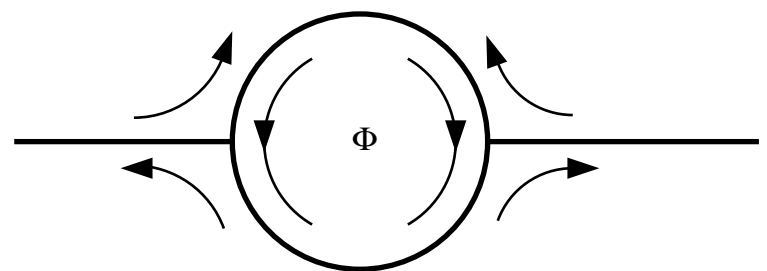

FIG. 1. An optimal quantum pump represented by a graph of ideal wires. For fixed value of the magnetic flux, $\Phi$, particles that enter from the left go clockwise around the loop and exit to the left, while particles that enter from the right go counterclockwise and exit to the right.

From Eq. (9), we see that increasing $\Phi$ by a unit of quantum flux draws one particle in from the right and expels one particle to the left. This is independent of the chemical potential $\mu$ of the bath, of the circumference $\ell$ of the loop, and of the position of the vertices on the loop.

At first glance the pumping expressed by Eq. (9) seems to be in conflict with common sense: The frozen $\mathrm{S}$ matrix allows for no transmission across the scatterer, so how can the pump transport any charge at all? This can be understood as follows. Increasing the flux creates an EMF around the loop that accelerates the clockwise-moving particles and decelerates the counterclockwise-moving particles. In particular, some of the low-energy counterclockwise-movers that entered from the right are turned around by the EMF and become clockwise-movers, after which they emerge to the left. This accounts for the net transfer of charge from right to left. Since the scattering at each vertex is deterministic, the outgoing channels have no entropy, the inequalities (7) become equalities, and the pump is optimal. A little reflection shows that Fig. I is a graph theoretic description of the quantum Hall effect. In the quantum Hall effect, time reversal is broken by an external magnetic field. In Fig. 1, time reversal is broken by the permutation matrix associated with the scattering condition at the vertices. More precisely, the vertex conditions at the vertices can be implemented by the edge currents in the quantum Hall effect in a Corbino disc [10].

Concluding remarks: We have analyzed quantum pumps as a problem in adiabatic scattering theory. We have focused on transport properties that admit instantaneous description, i.e. on times scales that are small compared to the pump cycle. In this theory a central role is played by the matrix of energy shift. We proved a general bound on the dissipation in quantum channels that motivated a notion of optimal quantum. Optimal quantum pumps: have geometric significance [11]; saturate a bound on dissipation; transport integer charges in closed cycles; are noiseless and minimize entropy production. Finally, we showed that the integer quantum Hall effect may be interpreted as an optimal pump.

Acknowledgment: We thank B. Altshuler, A. Kamenev, Y. Makhlin, M. Reznikov, and U. Sivan for discussion. The work was partially supported by the Israel Science Foundation and the Texas Advanced Research Program and the fund for the promotion of Research at the Technion and the NSF grant PHY-9971149.

[1] L. Eisenbud, Dissertation, Princeton University, 1948 (unpublished); E.P. Wigner, Phys. Rev. 98, 145 (1955). The time it takes a particle to traverse the scatterer gives a classical time scale. The quantum time scale $\tau$ is dictated by the level spacing of the disconnected scatterer. For one dimensional scatterers the two time scales coincide. If the scatterer is multidimensional the two time scales are in general different. Their ratio may be interpreted as the number of channels in the scatterer.

[2] M. Büttiker, H. Thomas, A. Prêtre, Z. Phys. B94, 133 (1994).

[3] P.W. Brouwer, Phys. Rev. B58, 10135 (1998); J. Avron, A. Elgart, G.M. Graf, L. Sadun, Phys. Rev. B62, R10618 (2000).

[4] L.S. Levitov and G.B. Lesovik, JETP Lett. 58461 (1993); D. A. Ivanov, H. W. Lee, and L. S. Levitov, Phys. Rev. B 56, 6839, (1997); L.S. Levitov, H. Lee and G.B. Lesovik, J. Math. Phys. 37, 4845, (1996); L.S. Levitov, cond-mat/0103617

[5] E.H. Lieb, M. Loss, Analysis, AMS (1997).

[6] A. Andreev and A. Kamenev, Phys. Rev. Lett. 85, 1294 (2000)

[7] B.A. Dubrovin, A.T. Fomenko, S.P. Novikov, Modern Geometry - Methods and Applications. Part II, Springer (1985).

[8] M. Switkes, C.M. Marcus, K. Campman, and A.G. Gossard, Science 283, 1907 (1999). It has been questioned if the correct interpretation of this experiment is indeed in terms of quantum pumps, see P. Brouwer, Phys. Rev. B 63, 121303 (2001).

[9] D.R. Yafaev, Mathematical Scattering Theory, AMS (1992).

[10] V.T. Dolgopolov, N.B. Zhitenev and A.A. Shacking, Pis'ma Zh. Ekp. Theor. Fiz. 52, 826 (1990).

[11] Shortly after the first version on this paper was posted on the archive, Y. Makhlin and A. Mirlin, condmat/0105414 derived interesting geometric results about noise in pumps. Some of their results overlap with some of ours. 